\title{
ON PRODUCTS OF MODULES IN A TOPOS
}

\author{
JAVAD TAVAKOLI \\ (Received 29 September 1982; revised 11 May 1983) \\ Communicated by R. H. Street
}

\begin{abstract}
In an elementary topos if $R$ is a ring and $X$ is a decidable object then there exists a canonical homomorphism from the coproduct of an $X$-family of $R$-modules to the product of the same family. In this paper it is shown that this homomorphisms is a monomorphism.
\end{abstract}

1980 Mathematics subject classification (Amer. Math. Soc.): primary 18 B 25; secondary 03 G 30.

In the category Set of sets if $X$ is a set and $R$ is a ring then for an $X$-family $\left\{M_{x}\right\}_{x \in X}$ of $R$-modules there is always a canonical monomorphism $\phi: \oplus_{x \in X} M_{x}$ $\rightarrow \Pi_{x \in X} M_{x}$, with $\pi_{x} \phi i_{x}=1_{M_{x}}$, where $\pi_{x}$ and $i_{x}$ are the $x$ th projection and injection, respectively. In [5] it is shown, by an example, that in an elementary topos such a homomorphism does not always exist. However, if we choose $X$, the index object, to be decidable, it is proved that such a canonical homomorphism exists.

In this paper we show that the canonical homomorphism given in [5] is a monomorphism. A closely related work can be found for the case of abelian groups in [1].

Throughout the paper, $E$ denotes an elementary topos with natural numbers object, and $R$ is a ring in $E$. All other notation, not explained here, can be found in [2] or [3].

Let $A$ be an $E$-indexed category with $\lim _{\rightarrow}$ and small homs, let $C$ be an internal category of $E$, let $\Gamma: C \rightarrow E$ be an internal functor with $\lim _{\rightarrow} \Gamma=I$ and for each $J$ in $E$ let $\Gamma^{J}(c) \stackrel{\lambda_{c}^{J}}{\rightarrow} J^{*} I$ be the canonical injection, where $c \in[J, C]$.

(C) 1985 Australian Mathematical Society $0263-6115 / 85 \$ A 2.00+0.00$ 
1. Lemma. If $A \in A^{I}$ then

$$
\sum_{I} A \simeq \underset{C}{\lim } \sum_{\Gamma^{J}(c)} \lambda_{c}^{J_{*}} \pi_{2}^{*} A,
$$

where $\pi_{2}: J \times I \rightarrow I$ is the projection.

Proof. Let $B \in A^{1}$. Then we have the following natural isomorphisms:

$$
\begin{gathered}
\lim _{C} \sum_{\Gamma^{J}(c)} \lambda_{c}^{J^{*}} \pi_{2}^{*} A \rightarrow B ; \\
\text { indexed cocone }\left\langle\sum_{\Gamma^{J}(c)} \lambda_{c}^{J^{*}} \pi_{2}^{*} A \rightarrow J^{*} B\right\rangle_{c \in[J, C]} \\
\text { compatible families }\left\langle\lambda_{c}^{J^{*}} \pi_{2}^{*} A \rightarrow \Gamma^{j}(c)^{*} J^{*} B\right\rangle_{c \in[J, C]} ; \\
\text { compatible families }\left\langle\lambda_{c}^{J_{*}} \pi_{2}^{*} A \rightarrow \lambda_{c}^{J^{*}} \pi_{2}^{*} I^{*} B\right\rangle_{c \in[J, C]} ; \\
\text { indexed cocone }\left\langle\pi_{2} \lambda_{c}^{J} \rightarrow \operatorname{Hom}^{I}\left(A, I^{*} B\right)\right\rangle_{c \in[J, C]} ; \\
1=\lim _{C}^{\rightarrow} \pi_{2} \lambda_{c}^{J} \rightarrow \operatorname{Hom}^{I}\left(A, I^{*} B\right) ; \\
A \rightarrow I^{*} B ; \\
\sum A \rightarrow B .
\end{gathered}
$$

Hence by the Yoneda Lemma,

$$
\underset{c}{\lim } \sum_{\Gamma^{J}(c)} \lambda_{c}^{J^{*}} \pi_{2}^{*} A \simeq \sum_{I} A .
$$

We will use the following theorem, due to D. Schumacher, which is proved in [4].

2. TheOREM. Let $A$ be a small filtered indexed category and let $F: A \rightarrow E$ be an indexed functor.

(a) For every $1 \stackrel{x}{\rightarrow} I^{*} \lim _{\rightarrow} F$ there exist $J \stackrel{\alpha}{\rightarrow} I, A \in A^{J}$ and $1 \stackrel{y}{\rightarrow} F^{J}(A)$ such that

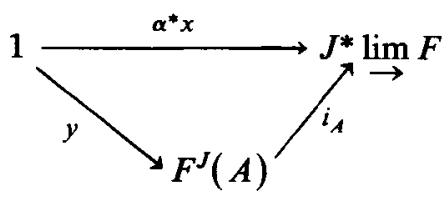

commutes, where $i_{A}$ is indexed. 
(b) For $I \stackrel{x_{1}}{\rightarrow} F^{I} A_{1}$ and $I \stackrel{x_{2}}{\rightarrow} F^{I} A_{2}, i_{A_{1}}\left(x_{1}\right)=i_{A_{2}}\left(x_{2}\right)$ if and only if there exist $J \stackrel{\alpha}{\longrightarrow} I$ and

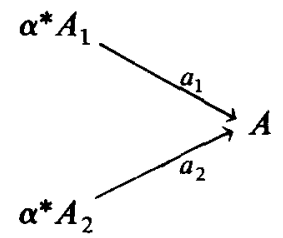

in $A^{J}$, such that $F^{J}\left(a_{1}\right)\left(\alpha^{*} x_{1}\right)=F^{J}\left(a_{2}\right)\left(\alpha^{*} x_{2}\right)$.

Let $X$ be an object in $E$ and let $E_{\text {fin }}$ be the internalization of $E_{\text {fc }}$, the category of finite cardinals, in the sense that its external category of $I$-elements is equivalent to $(E / I)_{\mathrm{fc}}$. For more details and the proof of the following lemma see [5] and [4].

3. LEMMA. The internal category $E_{\mathrm{fio}} / X$ is filtered.

Let $M$ be an object in $\operatorname{Mod}_{R}(E)^{X}$. Define a functor $P: E_{\mathrm{fin}} / X \rightarrow \operatorname{Mod}_{R}(E)$ as follows: for any $I$-object of $E_{\mathrm{fin}} / X$, i.e. $\left(I \stackrel{p}{\rightarrow} N,[p] \stackrel{x}{\rightarrow} I^{*} X\right), P^{I}\left([p] \stackrel{x}{\rightarrow} I^{*} X\right)=$ $\oplus_{[p]} x^{*} \pi_{2}^{*} M$, where $\pi_{2}: I \times X \rightarrow X$, and for any $I$-morphism

$$
\begin{gathered}
{[p] \stackrel{f}{\rightarrow}[q]} \\
\searrow \swarrow \\
I^{*} X
\end{gathered}
$$

$P^{I}(f)$ is defined by

$$
\begin{aligned}
{\left[\bigoplus_{[p]} x^{*} \pi_{2}^{*} M, L\right] } & \simeq\left[\pi_{2}^{*} M, \prod_{x}[p]^{*} L\right] \\
{\left[P^{\prime}(f), L\right] \uparrow } & \uparrow\left[\pi_{2}^{*} M, f^{*}\right] \\
{\left[\bigoplus_{[q]} y^{*} \pi_{2}^{*} M, L\right] } & \simeq\left[\pi_{2}^{*} M, \prod_{y}[q]^{*} L\right],
\end{aligned}
$$

where $L$ is in $\operatorname{Mod}_{R}(E)^{I}$. It is easy to see that $P$ is an indexed functor.

Let $I$ be a decidable object in $E$, i.e. $\delta: I \nrightarrow I \times I$, the diagonal morphism, has a complement $J \stackrel{c}{\rightarrow} I \times I$ such that $\left(\begin{array}{c}\delta \\ c\end{array}\right): I+J \rightarrow I \times I$ is an isomorphism. Then it is well known that $E / I \times I \stackrel{\left(\delta^{*}, c^{*}\right)}{\rightarrow} E / I \times E / J$ is an equivalence of categories. This extends to an equivalence $\alpha$ : $\operatorname{Mod}_{R}(E)^{I \times I} \rightarrow \operatorname{Mod}_{R}(E)^{I} \times \operatorname{Mod}_{R}(E)^{J}$. For $M$ in $\operatorname{Mod}_{R}(E)^{I}$, let $\alpha\left(\pi_{1}^{*} M\right)=\left(M_{1}, M_{1}^{\prime}\right)$ and $\alpha\left(\pi_{2}^{*} M\right)=\left(M_{2}, M_{2}^{\prime}\right)$, where $I \stackrel{\pi_{2}}{\rightarrow} I \times$ $I \stackrel{\pi_{1}}{\rightarrow} I$ are the projections and $M_{1}^{\prime}, M_{2}^{\prime}$ are in $\operatorname{Mod}_{R}(E)^{J}$. Since $\pi_{1} \delta=\pi_{2} \delta=1_{I}$, then $M_{1} \simeq M \simeq M_{2}$ in $\operatorname{Mod}_{R}(E)^{I}$, i.e. there is an isomorphism $M_{1} \stackrel{\theta}{\rightarrow} M_{2}$. Thus 
we have a morphism

$$
\alpha\left(\pi_{1}^{*} M\right)=\left(M_{1}, M_{1}^{\prime}\right) \stackrel{(\theta, 0)}{\rightarrow}\left(M_{2}, M_{2}^{\prime}\right)=\alpha\left(\pi_{2}^{*} M\right)
$$

and so there is a homomorphism $\psi: \pi_{1}^{*} M \rightarrow \pi_{2}^{*} M$, because $\alpha$ is an equivalence. By the Beck condition the canonical homomorphism $\gamma: \oplus_{\pi_{2}} \pi_{1}^{*} M \rightarrow I^{*} \oplus_{I} M$ is an isomorphism, and hence we have the following natural isomorphisms:

$$
\begin{array}{rlll}
\pi_{1}^{*} M & \stackrel{\psi}{\rightarrow} & \pi_{2}^{*} M & \text { in } \operatorname{Mod}_{R}(E)^{I \times I} ; \\
\bigoplus_{\pi_{2}} \pi_{1}^{*} M & \rightarrow & M & \text { in } \operatorname{Mod}_{R}(E)^{I} ; \\
I^{*} \bigoplus_{I} M & \stackrel{\bar{\phi}}{\rightarrow} & M & \text { in } \operatorname{Mod}_{R}(E)^{I} ; \\
\bigoplus_{I} M & \rightarrow & \prod_{I} M & \text { in } \operatorname{Mod}_{R}(E) .
\end{array}
$$

If $\eta^{\prime}$ is the unit for $\oplus_{\pi_{2}} \rightarrow \pi_{2}^{*}$ and $\varepsilon$ is the counit for $I^{*} \vdash \Pi_{I}$, then $\psi$ and $\phi$ are related by the equation $\left(\pi_{2}^{*} \bar{\phi}\right)\left(\pi_{2}^{*} \gamma\right) \eta_{\pi_{1}^{*} M}^{\prime}=\psi$, where $\bar{\phi}=\varepsilon_{M}\left(I^{*} \phi\right)$. On the other hand the canonical morphism $\gamma$ satisfies $\left(\pi_{2}^{*} \gamma\right) \eta_{\pi_{1}^{*} M}^{\prime}=\pi_{1}^{*} \eta_{M}$, where $\eta$ is the unit for $I^{*} \vdash \Pi_{I}$, so we have $\left(\pi_{2}^{*} \bar{\phi}\right)\left(\pi_{1}^{*} \eta_{M}\right)=\psi$. Apply $\delta^{*}$ to this equality to get $\bar{\phi} \eta_{M}=\delta^{*} \psi=1_{M}$ (by definition of $\psi$ ), i.e. $\varepsilon_{M}\left(I^{*} \phi\right) \eta_{M}=1_{M}$.

The main result of this paper is as follows.

4. Theorem. Let $X$ be a decidable object in $E$ and $M$ be an object in $\operatorname{Mod}_{R}(E)^{X}$. Then the homomorphism $\oplus_{X} M \stackrel{\phi}{\rightarrow} \Pi_{X} M$, defined above, is a monomorphism.

Proof. Let $T \stackrel{m}{\rightarrow} \oplus_{X} M$ be a $T$-element of $\oplus_{X} M$ such that $\phi(m)=0$. We have to show that $m=0$. By Lemma $1, \oplus_{X} M \simeq \lim _{c} \oplus_{[p]} x^{*} \pi_{2}^{*} M$, where $[p] \stackrel{x}{\rightarrow} I^{*} X$ is in $E / I$ and $C=E_{\mathrm{fin}} / X$. Apply the first part of Theorem 2 to the filtered indexed category $E_{\mathrm{fin}} / X$ and the indexed functor $P$, defined above (we can do that because filtered colimits in $\operatorname{Mod}_{R}(E)$ are the same as in $E$ ) to get $L \stackrel{\alpha}{\rightarrow}, p$ : $L \rightarrow N, x:[p] \rightarrow L^{*} X$, and $1 \rightarrow \oplus_{[p]} x^{*} \pi_{2}^{*} M$ such that $\alpha^{*} m=i_{x} y$, where $i_{x}$ : $P^{L}(x) \rightarrow L^{*} \lim _{\rightarrow} c^{P}$ is the indexed injection. But by the properties of colimit the following diagram commutes:

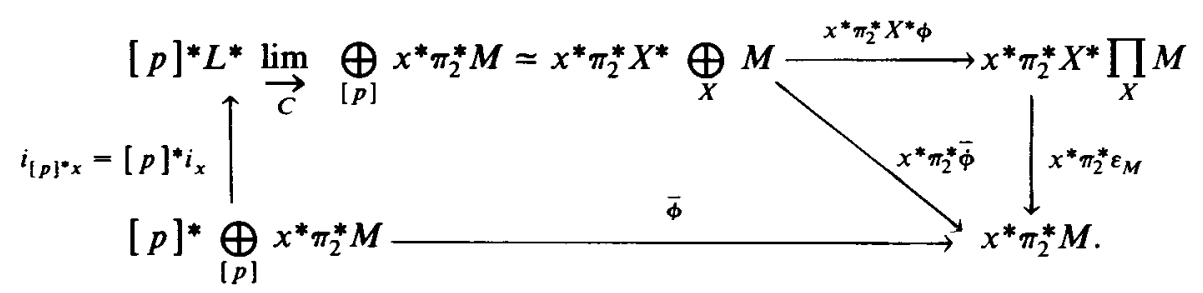


Since $x^{*} \pi_{2}^{*} X^{*}=[p]^{*} L^{*}$, then by transpose of the above diagram along $[p]^{*}$ we get

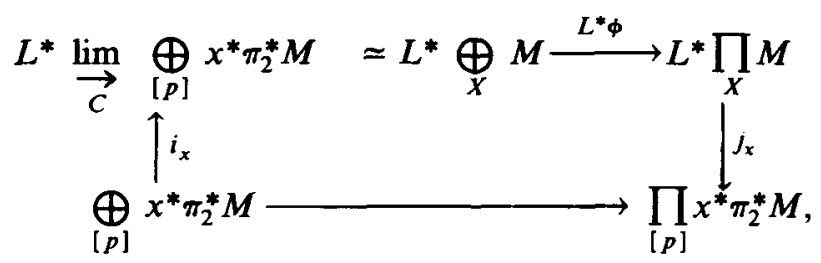

where $j_{x}$ is the transpose of $x^{*} \pi_{2}^{*} \varepsilon_{M}$. Now, by Theorem 2.3 [5],

$$
\bigoplus_{[p]} x^{*} \pi_{2}^{*} M \stackrel{\phi}{\rightarrow} \Pi_{[p]} x^{*} \pi_{2}^{*} M
$$

is an isomorphism. Therefore $j_{x} L^{*} \phi\left(\alpha^{*} m\right)=j_{x} L^{*} \phi\left(i_{x} y\right)=\phi(y)=0$ implies $y=$ 0 , so $\alpha^{*} m=0$. But $\alpha$ is an epimorphism and hence $m=0$, as required.

\section{References}

[1] R. Harting, 'Internal coproduct of Abelian groups in an elementary topos,' Comm. in Alg. 10(11), 1173-1237 (1982).

[2] P. Johnstone, Topos theory (L. M. S. Mathematical Monographs No. 10, Academic Press, 1977).

[3] R. Paré and D. Schumacher, 'Abstract families and the adjoint functor theorems' (Indexed Categories and their Application, Springer Lecture Notes in Math. 661 (1978)), 1-125.

[4] J. Tavakoli, Vector spaces in topoi, Ph.D., thesis, Dalhousie University, Canada (1980).

[5] J. Tavakoli, 'Elements of free modules in topoi', Comm. in Alg. 10(2) 171-201 (1982)

Department of Mathematics

Mashhad University

Mashhad

Iran 